Irish Math. Soc. Bulletin

Number 74, Winter 2014, 81-88

ISSN 0791-5578

\title{
MATTHEW O'BRIEN: AN INVENTOR OF VECTOR ANALYSIS
}

\author{
PETER LYNCH
}

\begin{abstract}
In the mid-nineteenth century, several mathematicians were engaged in a search for a new symbolism and methodology to express and solve physical problems in three dimensions. Hamilton's quaternions promised much but ultimately proved unequal to the task. Around 1880, the work of Gibbs and Heaviside led to the modern formulation of vector analysis. But decades earlier an essentially equivalent approach was formulated by Matthew O'Brien and applied to problems in geometry, statics, dynamics and optics. Although his work deserved greater attention, it was ignored by his contemporaries. One reason was that he was overshadowed by the towering and influential figure of Hamilton.
\end{abstract}

\section{INTRODUCTION}

Open any modern textbook on mechanics and bold letters will announce vector quantities. Vectors now play a central role in dynamics, fluid mechanics, elasticity and electromagnetism. Their popularity grew rapidly following the publication in the 1880s of Josiah Willard Gibbs' Elements of Vector Analysis, but there were many precursors of this work. The quaternions of William Rowan Hamilton promised much but were ill-suited to most problems in physics. In addition to Hamilton, another Irish mathematician contributed in a substantial way to vector analysis, namely Matthew O'Brien, the subject of this note.

\section{Matthew O'Brien}

Rev. Matthew O'Brien was one of several Irish mathematicians who flourished in Cambridge in the nineteenth century. Little is known about his personal life. He was born in Ennis, Co. Clare in 1814, where his father, also Matthew, was a medical doctor. He

2010 Mathematics Subject Classification. 01A55, 15-03, 15A72, $26 \mathrm{~B} 12$.

Key words and phrases. Vector Analysis, Mechanics, Biography.

Received on 29-8-2014; revised 13-11-2014. 


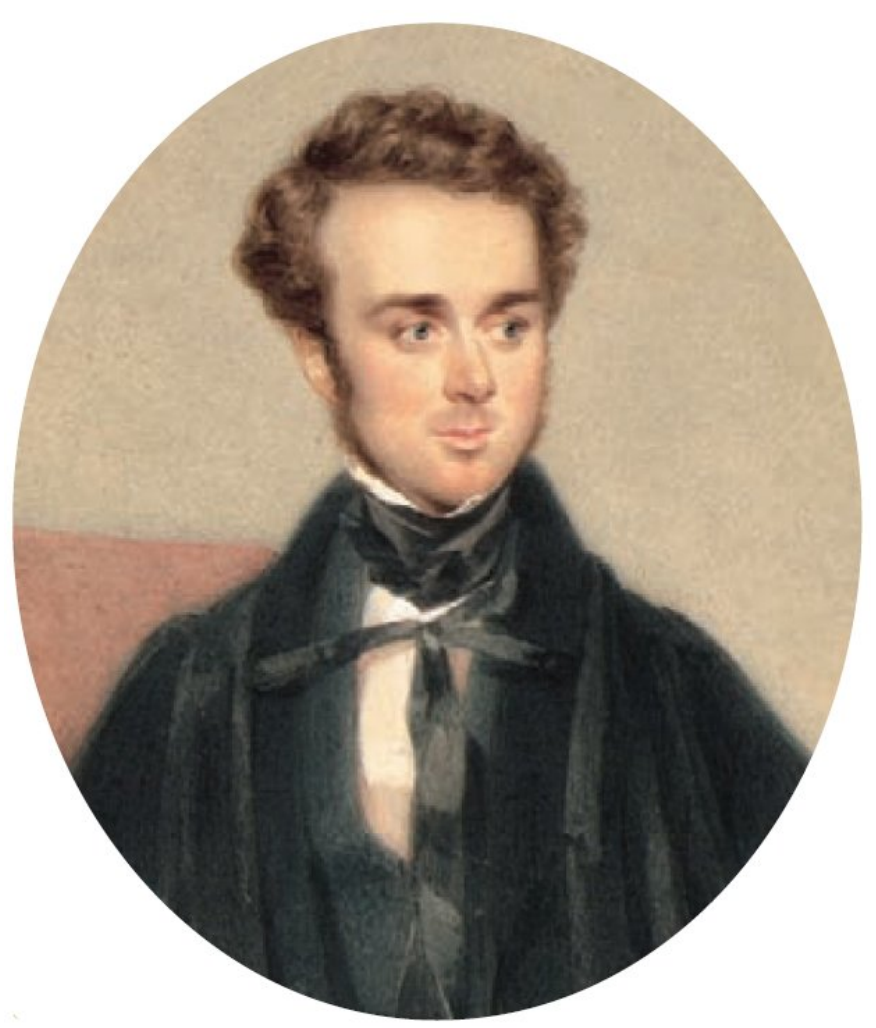

FiguRE 1. Matthew O'Brien (1814-1855). Illustration from Craik, 2008.

was ordained a clergyman of the Church of England. He had a son, Arthur Evanson O'Brien, who also graduated from Cambridge (in 1871) and who also took holy orders. Matthew O'Brien died in Jersey in 1855, while on a visit to recuperate from an illness.

In 1830 O'Brien entered Trinity College Dublin, aged just sixteen, and remained there until 1834. During his time in Trinity, he must have come into contact with William Rowan Hamilton, and was probably taught by him. Later, he became acquainted with Hamilton's work on quaternions, which generated widespread interest. Hamilton, whose attention from 1843 onwards was focussed almost exclusively on quaternions, made only the most fleeting reference to O'Brien in his published work. O'Brien made reference to Hamilton's work, most notably in his major paper of 1852. However, it is unclear just how much influence Hamilton's work had on O'Brien.

O'Brien was admitted to Gonville and Caius College Cambridge in 1834, aged 20. He was a pupil of the enormously effective tutor William Hopkins, who taught many of the leading mathematicians 
in Cambridge (Craik, 2008). Hopkins coached nearly 200 wranglers, of whom 17 were placed first in the entire university in the Mathematical Tripos, the final honours mathematics examinations, earning him the nick-name 'Senior Wrangler Maker'. In 1838 O'Brien was Third Wrangler, that is third place in the university. He was elected a Fellow of Caius College in 1840, and graduated as an MA the following year.

In 1844, O'Brien was appointed Professor of Natural Philosophy and Astronomy at King's College, London. From 1849 he also lectured in mathematics at the Royal Military Academy in Woolwich. In 1854 he resigned from King's to take up an appointment as Professor of Mathematics in Woolwich. Other applicants for the position at the Royal Military Academy included G. G. Stokes and J. J. Sylvester, the latter of whom was disgusted that he was not appointed. But Sylvester did not have long to wait. O'Brien died less than a year later, while recuperating in Jersey, and was succeeded at Woolwich by Sylvester.

\section{Vector Analysis}

O'Brien was the author of about twenty papers on mathematics. In addition, he published several books, including elementary texts on differential calculus and plane coordinate geometry and a treatise on mathematical geography. These books were good examples of expository writing and were moderately successful.

O'Brien's most notable contribution to mathematics was on the theory and application of vector methods. Hamilton's quaternions were cumbersome and were not convenient for applications. In quaternion algebra, if $q_{1}=\mathbf{V}_{1}$ and $q_{2}=\mathbf{V}_{2}$ are two quaternions with vanishing scalar parts, their product is

$$
q_{1} q_{2}=-\mathbf{V}_{1} \cdot \mathbf{V}_{2}+\mathbf{V}_{1} \times \mathbf{V}_{2} .
$$

Thus, the scalar and vector products are inherent components of Hamilton's quaternions, but they are conflated into a single operation. Simpler, more suitable, methods were widely sought. Vectors have two multiplication operators, the vector and scalar products, which make them eminently suitable for application to a wide range of problems in physics. Ultimately, vectors proved invaluable in giving concise expression to the equations of fluid mechanics, electromagnetism and elasticity. 
O'Brien's evolving ideas on vector formulations of mechanics were published in a series of seven papers between 1847 and 1852. A full list of relevant publications is given in Crowe (1967, p. 108, Note 92). O'Brien anticipated many of the results that appeared later in Gibbs' Vector Analysis. His treatment of vectors was remarkably complete. O'Brien defined the scalar and vector products for vector quantities. His notation can be confusing: he wrote $u \times v$ for the scalar (or dot) product and $u \cdot v$ or $D u \cdot v$ for the vector (or cross) product, essentially the reverse of the modern convention. He showed that the vector product is non-commutative and he derived several vector identities that are now standard.

O'Brien also defined the gradient operator; Hamilton had introduced this operator earlier and, presumably, O'Brien was aware of this although he did not explicitly acknowledge Hamilton in this context. His papers include identities such as

$$
\text { curl curl } \mathbf{V}=\operatorname{grad} \operatorname{div} \mathbf{V}-\operatorname{div} \operatorname{grad} \mathbf{V}
$$

although, of course, his notation was quite different. Indeed, O'Brien derived almost all the substantive results that appear in the initial chapters of Gibbs' Vector Analysis. He expressed the basic equations of mechanics in vector form and applied them to problems such as computing the Earth's precession and nutation, deriving an expression for the annual precession of the polar axis.

The history of vector analysis is recounted in considerable detail by Crowe (1967). One thing that O'Brien missed was the lack of associativity of the vector product:

$$
\left(\mathbf{V}_{1} \times \mathbf{V}_{2}\right) \times \mathbf{V}_{3} \neq \mathbf{V}_{1} \times\left(\mathbf{V}_{2} \times \mathbf{V}_{3}\right)
$$

Crowe put special emphasis on this omission. However, it is hard to doubt that O'Brien was well aware of it as, in the case where $\mathbf{V}_{1}=\mathbf{V}_{2}$, the left hand side must vanish whereas the right hand side need not. It may be that O'Brien just took this for granted, much as we take for granted the lack of associativity for subtraction and division:

$$
(a-b)-c \neq a-(b-c) \quad \text { and } \quad(a \div b) \div c \neq a \div(b \div c) .
$$

For example, O'Brien wrote the equivalent, in his notation, of $(\mathbf{U} \times)^{2} \mathbf{V}$ which, if interpreted as $(\mathbf{U} \times \mathbf{U}) \times \mathbf{V}$ would vanish, but it is clear from the context that he intended $\mathbf{U} \times(\mathbf{U} \times \mathbf{V})$. 
In his review of O'Brien's work on vectors, Crowe (1967) recognized him as a forerunner of Gibbs and Heaviside. In some of his papers, O'Brien back-tracked, reverting to "the ordinary notation of algebra". However, in the 1852 paper he returned again to his novel vector methods. Crowe paid most attention to O'Brien's last paper (O'Brien, 1852), making only passing mention of his earlier work on vectors. Smith (1982) rectified this, reconsidering the earlier work in more detail.

Crowe quoted C. G. Knott, an enthusiast of quaternions who, in 1892, wrote that "Gibbs and Heaviside had barely advanced beyond the stage reached by O'Brien [in 1852]". Crowe observed that "if Knott was correct, then O'Brien deserves great credit and must be called the father of modern vector analysis". As already stated, Crowe's discussion focussed on O'Brien's 1852 paper, but Smith's later analysis of the earlier papers suggests that O'Brien went farther than indicated by Crowe, coming very close to modern vector analysis.

\section{The Rotating Earth}

O'Brien presented an interesting application of his vector method in his 1852 paper. A year prior to its publication, Léon Foucault's pendulum experiment in Paris had generated widespread interest. O'Brien considered the equations of motion on the rotating Earth. He wrote down the expression for the rate of change of a vector quantity in the non-inertial Earth frame:

$$
\left(\frac{\mathrm{d} \mathbf{A}}{\mathrm{d} t}\right)_{\mathrm{A}}=\left(\frac{\mathrm{d} \mathbf{A}}{\mathrm{d} t}\right)_{\mathrm{R}}+\Omega \times \mathbf{A}
$$

(we use modern notation but the expression is completely equivalent to O'Brien's equation). Here subscript A denotes the absolute frame and $\mathrm{R}$ the rotating frame, $\mathrm{A}$ is an arbitrary vector and $\Omega$ is the angular velocity of Earth. This appears to be the first occurrence of this important relationship in vector form. As it has no name, and is so central in geophysics, it would seem appropriate to call it O'Brien's equation.

O'Brien used the relationship (1) in the usual way, first applying it to the position vector $\mathbf{R}$ to get the link between the relative and absolute velocity,

$$
\mathbf{V}_{\mathrm{A}}=\mathrm{V}_{\mathrm{R}}+\Omega \times \mathbf{R}
$$


and then to the velocity to get the acceleration

$$
\left(\frac{\mathrm{d} \mathbf{V}}{\mathrm{d} t}\right)_{\mathrm{A}}=\left(\frac{\mathrm{d} \mathbf{V}}{\mathrm{d} t}\right)_{\mathrm{R}}+2 \boldsymbol{\Omega} \times \mathbf{V}+\boldsymbol{\Omega} \times(\boldsymbol{\Omega} \times \mathbf{R})
$$

O'Brien realized that the centrifugal acceleration, the last term in the above equation, could be combined with Newtonian gravitation, so that the final vector equation for motion relative to the Earth becomes

$$
\frac{\mathrm{d} \mathbf{V}}{\mathrm{d} t}=-2 \boldsymbol{\Omega} \times \mathbf{V}+\mathbf{F}
$$

(subscript $\mathrm{R}$ now omitted) where $\mathbf{F}$ is the sum of the applied forces (per unit mass). The term involving $\Omega$ is what we now call the Coriolis term. O'Brien was the first person to express the Coriolis term in vector form. He described the term $-2 \Omega \times \mathbf{V}$ as "the force which must be supposed to act as a correction for the neglected rotation [in the non-inertial frame]".

O'Brien gave a complete solution for the motion of the pendulum on the rotating Earth, showing that the period is inversely proportional to the sine of latitude. He also gave an interesting interpretation of the motion as the super-position of two conical motions with slightly different frequencies. All in all, this was an impressive demonstration of the vector method in analysing a problem of great topical interest.

\section{Overshadowed}

O'Brien came quite close to constructing the system of vector algebra as it is used today. Yet, despite his innovative work, his ideas were almost completely ignored by his contemporaries, and it was several decades before Gibbs' Vector Analysis lit a bright flame. One of the reasons was that Hamilton was a figure of towering influence and he and his supporters worked indefatigably to promote the recognition and use of quaternions. O'Brien's work was completely overshadowed by this 'publicity campaign'.

O'Brien might have achieved much more had he had more leisure to pursue his research. But he was overburdened with teaching responsibilities, and his life was cut short at just forty-one years. While his formulation of vector analysis was incomplete, and imperfect in some respects, it merits recognition as a significant contribution. Designating any individual as the "father of modern vector analysis" is problematical. We must also recognise that at the time 
that O'Brien was beginning his work on vectors, Hermann Grassmann published his Ausdehnungslehre (Grassmann, 1844), a profound work of sweeping generality embracing many of the crucial concepts that underlie vector analysis.

We can conclude that O'Brien deserves more credit that he received in his short life. One means of rectifying this lack of recognition would be to give a new designation to the expression (1) for the rate of change of a vector quantity in a non-inertial frame. This equation plays a central role in geophysics, yet it has no accepted name. It is sometimes called Coriolis' Theorem, but this is inappropriate: while the physical content of the equation was well known to Coriolis, and the equation had been expressed in component form much earlier, O'Brien (1852, p. 193) was the first to formulate it as a vector equation. It would seem appropriate to call (1) O'Brien's equation.

\section{REFERENCES}

[1] Craik, Alex, D. D., 2008: Mr Hopkins' Men: Cambridge Reform and British Mathematics in the 19th Century. Springer, 405pp. ISBN: 9-781-848-00132-9.

[2] Crowe, M. J., 1967: A History of Vector Analysis: The Evolution of the Idea of a Vectorial System. Univ. Notre Dame Press. Reprinted by Dover, 1985/1994. Paperback edition with a new preface (New York: Dover, 1985); another edition with new introductory material (New York: Dover, 1994). ISBN: 9780-486-67910-5.

[3] Crowe, M. J., 2008: A History of Vector Analysis. Lecture based on Crowe (1967/1985/1994) [PDF online].

[4] Gibbs, Josiah Willard, 1881: Elements of Vector Analysis. Yale University, New Haven.

[5] Grassmann, Hermann, 1844: Die lineale Ausdehnungslehre, ein neuer Zweig der Mathematik (The Theory of Linear Extension, a New Branch of Mathematics). Leipzig: Wiegand. English translation: A New Branch of Mathematics, by Lloyd Kannenberg, Chicago: Open Court, 1995.

[6] Hayes, Cathy, 2009: Matthew O'Brien. Dictionary of Irish Biography, Ed. James McGuire and James Quinn, Royal Irish Academy and Camb. Univ. Press, Vol. 7, p. 63.

[7] O'Brien, M, 1852: On symbolic Forms derived from the Conception of the Translation of a Directed Magnitude, Phil. Trans. Roy. Soc. London, 142, 161-206.

[8] Platts, Charles, 1917: Matthew O'Brien. Dictionary of National Biography, Vol. 14, p. 764. Oxford Univ. Press.

[9] Smith, G. C., 1982: Matthew O'Brien's anticipation of vectorial mathematics. Hist. Math., 9, 172-190. 
Peter Lynch is Professor of Meteorology at UCD. His interests include dynamic meteorology, numerical weather prediction and Hamiltonian mechanics. He writes an occasional mathematics column That's Maths in the Irish Times; see his blog at http://thatsmaths. com.

School of Mathematical Sciences, University College Dublin E-mail address: Peter.Lynch@ucd.ie 